FedUni ResearchOnline

http://researchonline.federation.edu.au

This is an Accepted Manuscript of an article published by Taylor \& Francis in Optimization on 4/01/2016, available online: 


\title{
A Generalization of a Theorem of Arrow, Barankin and Blackwell to a Nonconvex Case
}

\author{
Refail Kasimbeyli* and Musa Mammadov**
}

October 3, 2012

\begin{abstract}
The paper presents a generalization of a known density theorem of Arrow, Barankin, and Blackwell for properly efficient points defined as support points of sets with respect to monotonically increasing sublinear functions. This result is shown to hold for nonconvex sets of a reflexive Banach space partially ordered by a Bishop-Phelps cone.
\end{abstract}

Key Words: vector optimization, ABB theorem, Bishop-Phelps cone, separation theorem, sublinear support functionals, augmented dual cone, proper efficiency.

\section{Introduction}

In 1953, Arrow, Barankin and Blackwell [1] proved a famous theorem (ABB theorem for short), concerning the density of the set of minimal solutions of strictly positive support functionals in the set of minimal elements of a compact convex subset $S$ in $\mathbb{R}^{n}$. This theorem has been the subject of many investigations and has been proved in various settings and under a variety

*Department of Industrial Engineering, Faculty of Engineering, Anadolu University, Eskisehir, Turkey, email: rkasimbeyli@anadolu.edu.tr

${ }^{* *}$ Centre for Informatics and Applied Optimization, University of Ballarat, Victoria, 3353, Australia, email: m.mammadov@ballarat.edu.au 
of assumptions. Borwein [5, 6], Hartley [15], and Bitran and Magnanti [4] proved density theorems in a finite-dimensional space for convex sets. Henig [16] extended this result to nonconvex sets in $\mathbb{R}^{n}$.

Radner [25] and Majumdar [21] generalized the Arrow-Barankin-Blackwell result the case of $l_{\infty}$. Majumdar [22] further extended this result to $L_{\infty}$. Salz [26] extended this theorem to real normed spaces partially ordered by a convex cone with a base norm, Borwein [7] presented a special version in a normed space with a weakly compact based cone, and Jahn showed that it remains true for convex sets in a real normed space partially ordered by a Bishop-Phelps cone [17]. The density theorems under convexity assumption was given also by Zheng [31], Woo and Goodrich [28], Ng and Zheng [24]. For a deeper discussion of existing results and the tradeoff between assumptions on the set and the cone we refer the reader to Ferro [9], Truong [27], and Woo and Goodrich [28].

In the case where $\mathbb{S}$ is not convex, the density result has been obtained for the set of Henig proper efficient points [2, 10, 23, 27, 14] and the set of superefficient points [8, 29, 30,32].

Most authors exploited the assumption of compactness or weak compactness of $\mathbb{S}[8,11,23,25,32]$. By requiring a normality assumption on the ordering cone $\mathbb{C}$, Truong weakened the compactness assumption on $\mathbb{S}$ and developed a technique based on the concept of Luc cone compactness to obtain existence and density results for proper efficiency [27]. Gopfert et al. [14] generalized the Henig's result to normed spaces for asymptotically compact sets $\mathbb{S}$ by assuming $\mathbb{S}_{\infty} \cap \mathbb{C}=\{0\}$, where $\mathbb{S}_{\infty}$ denotes the asymptotic cone of $\mathbb{S}$, and both asymptotically compactness and asymptotic cone are defined with respect to a locally convex topology. In both works [14, 27] ordering cone $\mathbb{C}$ is required to have a bounded base.

The aim of this paper is to present a density result for the special kind of properly efficient points of Hartley cone compact sets in reflexive Banach spaces partially ordered by a Bishop-Phelps cone. These properly efficient points were firstly investigated in [18] (see also [12, 19]) and defined as support points of sets with respect to monotonically increasing sublinear functions. This enables to use the supporting points of sets without convexity assumptions. The proper efficiency introduced by this way is shown to be equivalent to Benson's and Henig's proper efficiency in reflexive Banach spaces. In these investigations, the special separation property and the nonlinear separation theorem proved in [18] play an important role. In this paper we present a general sufficient condition for two cones to satisfy the separa- 
tion property. We prove also that this condition is necessary and sufficient for a cone to be a Bishop-Phelps cone.

The paper is organized as follows. Section 2 gives some preliminaries. In this section we also present the class of monotonically increasing functions and characterizations of properly efficient points defined with the help of these functions. Separation property and separation theorems are given in section 3. In this section a sufficient condition for separation property and a characterization of Bishop- Phelps cones in the form of necessary and sufficient conditions are also presented. The main theorem is given in section 4 . Finally, section 5 draws some conclusions from the paper.

\section{Preliminaries}

In this section, we recall some concepts of cones, separability and proper efficiency. Throughout the paper, we will assume always, unless stated specifically otherwise, that:

(i) $\mathbb{X}$ is a reflexive Banach space with dual space $\mathbb{X}^{*}$;

(ii) $\mathbb{S}$ is a nonempty subset of $\mathbb{X}$;

(iii) $\mathrm{cl}(\mathbb{S}), \operatorname{bd}(\mathbb{S})$, int $(\mathbb{S})$, and $\mathrm{co}(\mathbb{S})$ denote the closure (in the norm topology), the boundary, the interior, and the convex hull of a set $\mathbb{S}$, respectively;

(iv) $\mathbb{R}_{+}$denotes the set of nonnegative real numbers;

The unit sphere and unit ball of $\mathbb{X}$ are denoted by

$$
\mathbb{U}=\{x \in \mathbb{X}:\|x\|=1\}
$$

and

$$
\mathbb{B}=\{x \in \mathbb{X}:\|x\| \leq 1\}
$$

respectively.

A nonempty subset $\mathbb{C}$ of $\mathbb{X}$ is called a cone if

$$
x \in \mathbb{C}, \lambda \geq 0 \Rightarrow \lambda x \in \mathbb{C} .
$$


Pointedness of $\mathbb{C}$ means that

$$
\begin{gathered}
\mathbb{C} \cap(-\mathbb{C})=\left\{0_{\mathbb{X}}\right\} . \\
\text { cone }(\mathbb{S})=\{\lambda s: \lambda \geq 0 \text { and } s \in \mathbb{S}\} .
\end{gathered}
$$

denotes the cone generated by a set $\mathbb{S}$.

$\mathbb{C}_{\mathbb{U}}=\mathbb{C} \cap \mathbb{U}=\{x \in \mathbb{C}:\|x\|=1\}$ denotes the norm-base of the cone $\mathbb{C}$. The term norm-base is justified by the obvious assertion that $\mathbb{C}=\operatorname{cone}\left(\mathbb{C}_{\mathbb{U}}\right)$.

$\mathbb{S}$ is said to be Luc $\mathbb{C}-$ compact if any cover of $\mathbb{S}$ of the form $\left\{U_{\alpha}+\mathbb{C}\right.$ : $\alpha \in I, U_{\alpha}$ are open $\}$ admits a finite subcover; see [20, page 14].

$\mathbb{S}$ is said to be Hartley $\mathbb{C}-$ compact if, for any $x \in \mathbb{S}, \mathbb{S} \cap(\{x\}-\mathbb{C})$ is compact, see [15].

$\mathbb{S}$ is said to be $\mathbb{C}$-closed if $\mathbb{S}+\mathbb{C}$ is closed.

$\mathbb{S}$ is said to be $\mathbb{C}$-bounded if there is a bounded set $M \subset \mathbb{X}$ such that $\mathbb{S} \subseteq M+\mathbb{C}$.

For $x^{*} \in X^{*}$ and a real number $\alpha>0$, Bishop-Phelps cone is defined as

$$
\mathbb{C}\left(x^{*}, \alpha\right)=\left\{x \in \mathbb{X}: x^{*}(x) \geq \alpha\|x\|\right\} .
$$

Recall that the dual cone $\mathbb{C}^{*}$ of $\mathbb{C}$ and its quasi-interior $\mathbb{C}^{\#}$ are defined by

$$
\mathbb{C}^{*}=\left\{x^{*} \in \mathbb{X}^{*}: x^{*}(x) \geq 0 \text { for all } x \in \mathbb{C}\right\}
$$

and

$$
\mathbb{C}^{\#}=\left\{x^{*} \in \mathbb{X}^{*}: x^{*}(x)>0 \text { for all } x \in \mathbb{C} \backslash\{0\}\right\},
$$

respectively.

The following three cones called augmented dual cones of $\mathbb{C}$ were introduced in [18].

$$
\begin{array}{r}
\mathbb{C}^{a *}=\left\{\left(x^{*}, \alpha\right) \in \mathbb{C}^{\#} \times \mathbb{R}_{+}: x^{*}(x)-\alpha\|x\| \geq 0 \text { for all } x \in \mathbb{C}\right\}, \\
\mathbb{C}^{a \circ}=\left\{\left(x^{*}, \alpha\right) \in \mathbb{C}^{\#} \times \mathbb{R}_{+}: x^{*}(x)-\alpha\|x\|>0 \text { for all } x \in \operatorname{int}(\mathbb{C})\right\},
\end{array}
$$

and

$$
\mathbb{C}^{a \#}=\left\{\left(x^{*}, \alpha\right) \in \mathbb{C}^{\#} \times \mathbb{R}_{+}: x^{*}(x)-\alpha\|x\|>0 \text { for all } x \in \mathbb{C} \backslash\{0\}\right\},
$$

where $\mathbb{C}$ is assumed to have a nonempty interior in the definition of $\mathbb{C}^{a \circ}$.

Let $\mathbb{X}$ be partially ordered by a cone $\mathbb{C}$. 
Definition 2.1. (a) An element $\bar{x} \in \mathbb{S}$ is called a minimal element of $\mathbb{S}$ if $(\{\bar{x}\}-\mathbb{C}) \cap \mathbb{S}=\{\bar{x}\}$.

(b) Let $\operatorname{int}(\mathbb{C}) \neq \emptyset$. An element $\bar{x} \in \mathbb{S}$ is called a weakly minimal element of $\mathbb{S}$ if $(\{\bar{x}\}-\operatorname{int}(\mathbb{C})) \cap \mathbb{S}=\emptyset$.

(c) An element $\bar{x} \in \mathbb{S}$ is called a properly minimal element of $\mathbb{S}$ (in the sense of Benson [3]) if $\bar{x}$ is a minimal element of $\mathbb{S}$ and the zero element of $\mathbb{X}$ is a minimal element of the radial cone $R(\mathbb{S}+\mathbb{C}, \bar{x})$ of $(\mathbb{S}+\mathbb{C})$ at $\bar{x}$, where $R(\mathbb{S}+\mathbb{C}, \bar{x})=\operatorname{cl}($ cone $(\mathbb{S}+\mathbb{C}-\{\bar{x}\}))$.

(d) An element $\bar{x}$ is called a properly minimal element of $\mathbb{S}$ (in the sense of Henig [16]) if it is a minimal element of $\mathbb{S}$ with respect to some convex cone $\mathbb{K}$ with $\mathbb{C} \backslash\left\{0_{\mathbb{X}}\right\} \subset \operatorname{int}(\mathbb{K})$.

\subsection{The Class of Monotone Sublinear Functions}

We begin this section by recalling definitions of monotone functions.

Definition 2.2. Let $\mathbb{C}$ be a convex cone in a real normed space $(\mathbb{Y},\|\cdot\|)$, and let $g: \mathbb{Y} \rightarrow \mathbb{R}$ be a given function on $\mathbb{Y}$.

(a) The function $g$ on $\mathbb{Y}$ is called monotonically increasing if, for each $y_{1}, y_{2} \in \mathbb{Y}, y_{1}-y_{2} \in C \Rightarrow g\left(y_{1}\right) \geq g\left(y_{2}\right)$.

(b) The function $g$ on $\mathbb{Y}$ is called strongly monotonically increasing if, for each $y_{1}, y_{2} \in \mathbb{Y}, y_{1}-y_{2} \in C \backslash\{0\} \Rightarrow g\left(y_{1}\right)>g\left(y_{2}\right)$.

(c) If $\operatorname{int} C \neq \emptyset$, then the function $g$ on $\mathbb{Y}$ is called strictly monotonically increasing if, for each $y_{1}, y_{2} \in \mathbb{Y}, y_{1}-y_{2} \in \operatorname{int} C \Rightarrow g\left(y_{1}\right)>g\left(y_{2}\right)$.

The assertions of the following theorem were proved in [18]).

Theorem 2.3. Let $(\mathbb{Y},\|\cdot\|)$ be a real normed space partially ordered by a pointed closed convex cone $\mathbb{C}$. Let $y^{*} \in \mathbb{Y}^{*} \backslash\{0\}$ and $\alpha \in \mathbb{R}_{+}$, and let a function $g_{\left(y^{*}, \alpha\right)}: \mathbb{Y} \rightarrow \mathbb{R}$ be defined as

$$
g_{\left(y^{*}, \alpha\right)}(y)=y^{*}(y)+\alpha\|y\| .
$$

Then, the function $g_{\left(y^{*}, \alpha\right)}$ is monotonically increasing, strictly monotonically increasing (if $\operatorname{int}(\mathbb{C}) \neq \emptyset$ ), and strongly monotonically increasing on $\mathbb{Y}$ if and only if $\left(y^{*}, \alpha\right) \in \mathbb{C}^{a *},\left(y^{*}, \alpha\right) \in \mathbb{C}^{a \circ}$, and $\left(y^{*}, \alpha\right) \in \mathbb{C}^{a \#}$, respectively. 


\subsection{Characterization of Properly Minimal Elements}

In this section we introduce a new kind of properly minimal elements by using the class of monotonically increasing sublinear functions presented in the previous section. We define properly minimal elements as support points of a set with respect to the given class of functions. and prove that, Benson and Henig proper efficiencies both are equivalent to the presented one.

Definition 2.4. Let $\mathbb{S}$ be a nonempty subset of $\mathbb{X}$. An element $\bar{x} \in \mathbb{S}$ is called a properly minimal element of $\mathbb{S}$, if there exists a pair $\left(x^{*}, \alpha\right) \in \mathbb{C}^{a \#}$ such that

$$
x^{*}(\bar{x})+\alpha\|\bar{x}\| \leq x^{*}(x)+\alpha\|x\| \text { for all } x \in \mathbb{S} .
$$

The following assumption will be used throughout the paper to simplify the formulation of the separation property.

Assumption 2.5. Let $\mathbb{C}$ be a convex cone in a normed space $(\mathbb{X},\|\cdot\|)$. There exists a pair of elements $\left(x^{*}, \alpha\right) \in \mathbb{X}^{*} \times \mathbb{R}$ with $\alpha>0$ such that

$$
c l\left(\operatorname{co}\left(\mathbb{C}_{\mathbb{U}}\right)\right)=\left\{x \in \mathbb{B}: x^{*}(x) \geq \alpha\right\} .
$$

The following theorem shows that under certain conditions in reflexive Banach spaces the two definitions of proper minimality given by Benson and Henig, are equivalent. The similar theorem is proved in [18], where the ordering cone is assumed to have weakly compact base. The following theorem shows that the same assertions can be formulated in a more simpler form for ordering cones satisfying Assumption 2.5, without any conditions on the base (see [18, Theorem 5.8]).

Theorem 2.6. Let $\left(\mathbb{X},\|\cdot\|_{\mathbb{X}}\right)$ be a reflexive Banach space partially ordered by a closed cone $\mathbb{C}$ satisfying Assumption 2.5. Let $\mathbb{S}$ be a nonempty subset of $\mathbb{X}$. Then, the three definitions of properly efficient points given by Benson, by Henig and in Definition 2.4 are equivalent.

We omit the proof of this theorem because it follows from Theorem 5.8 given in [18]. 


\section{Separation Property and Characterization of Bishop-Phelps Cones}

In this section, we present the separation property introduced in [18]. The generalization of $\mathrm{ABB}$ theorem will be given for the sets in reflexive Banach spaces partially ordered by closed convex cones satisfying the separation property. This property enables to separate two cones (one of them is not necessarily convex, having only the vertex in common) by a level set of some monotonically increasing (with respect to the ordering cone) function. A general sufficient condition for two cones to satisfy the separation property is also presented in this section.

Definition 3.1. Let $\mathbb{C}$ and $\mathbb{K}$ be closed cones of a normed space $(\mathbb{X},\|\cdot\|)$ with norm-bases $\mathbb{C}_{\mathbb{U}}$ and $\mathbb{K}_{\mathbb{U}}$, respectively. Let $\mathbb{K}_{\mathbb{U}}^{\partial}=\mathbb{K}_{\mathbb{U}} \cap$ bd $(\mathbb{K})$, and let $\widetilde{\mathbb{C}}$ and $\widetilde{\mathbb{K}}^{\partial}$ be the closures of the sets $\operatorname{co}\left(\mathbb{C}_{\mathbb{U}}\right)$ and co $\left(\mathbb{K}_{\mathbb{U}}^{\partial} \cup\left\{0_{\mathbb{X}}\right\}\right)$, respectively. The cones $\mathbb{C}$ and $\mathbb{K}$ are said to have the separation property with respect to the norm $\|\cdot\|$ if

$$
\widetilde{\mathbb{C}} \cap \widetilde{\mathbb{K}}^{\partial}=\emptyset
$$

Definition 3.2. Let $\mathbb{C}$ and $\mathbb{K}$ be nonempty cones of a normed space $(\mathbb{X},\|\cdot\|)$ with int $(\mathbb{K}) \neq \emptyset$. A cone $\mathbb{K}$ is called a conic neighborhood of $\mathbb{C}$ if $(\mathbb{C} \backslash$ $\left.\left\{0_{\mathbb{X}}\right\}\right) \subset \operatorname{int}(\mathbb{K})$. For a positive real number $\varepsilon$, a cone $\mathbb{C}_{\varepsilon}=$ cone $\left(\mathbb{C}_{\mathbb{U}}+\varepsilon \mathbb{B}\right)$ is called an $\varepsilon$-conic neighborhood of $\mathbb{C}$.

The following lemma presents a general sufficient condition for the separation property.

Lemma 3.3. Let $\mathbb{C}$ and $\mathbb{K}$ be two given closed cones in a reflexive Banach space $(\mathbb{X},\|\cdot\|)$ such that, $\mathbb{C}$ is a convex cone for which Assumption 2.5 is satisfied, and $\mathbb{C} \cap \mathbb{K}=\{0\}$. Then $\mathbb{C}$ and $\mathbb{K}$ satisfy the separation property given in Definition 3.1. 
Proof. Let $\widetilde{\mathbb{C}}$ and $\widetilde{\mathbb{K}}^{\partial}$ be as in Definition 3.1. We will show that

$$
\widetilde{\mathbb{C}} \cap \widetilde{\mathbb{K}}^{\partial}=\emptyset \text {. }
$$

Let $\left(x^{*}, \alpha\right) \in X^{*} \times \mathbb{R}$ with $\alpha>0$ be the given pair of elements in condition (9).

It is not difficult to show that

$$
x^{*}(x)<\alpha, \quad \forall x \in \mathbb{K}_{U} .
$$

Indeed, if $x^{*}(x) \geq \alpha$ for some $x \in \mathbb{K}_{U}$, then from (9) it follows $x \in \mathbb{C}_{U} \subset \mathbb{C}$ that contradicts to $\mathbb{C} \cap \mathbb{K}=\{0\}$.

Due to the reflexivity of $\mathbb{X}$, the unit ball $\mathbb{B}$ of this space is weakly compact. Then $\mathbb{K}_{U}$ becomes weakly compact as a closed subset of weakly compact set. Then there exists a positive number $\alpha^{\prime}<\alpha$ such that $x^{*}(x) \leq \alpha^{\prime}$ for all $x \in \mathbb{K}_{U}$. This inequality holds also for $x=\mathbf{0}$. Then, it is satisfied for all $x \in \mathbb{K}_{U} \cup\{\mathbf{0}\}$. Therefore, we obtain that

$$
x^{*}(x) \leq \alpha^{\prime}, \quad \forall x \in \operatorname{co}\left(\mathbb{K}_{\mathrm{U}} \cup\{\mathbf{0}\}\right),
$$

and, in particular,

$$
x^{*}(x) \leq \alpha^{\prime}, \quad \forall x \in \widetilde{K}^{\partial}=\operatorname{cl}\left(\operatorname{co}\left(\mathbb{K}_{\mathrm{U}}^{\partial} \cup\{\mathbf{0}\}\right)\right)
$$

Finally, taking into account the fact that, $\operatorname{cl}\left(\operatorname{co}\left(\mathbb{K}_{\mathrm{U}}^{\partial} \cup\{\mathbf{0}\}\right)\right) \subset \mathbb{B}$, from (9) and (12) we obtain $\widetilde{C} \cap \widetilde{K}^{\partial}=\emptyset$, which completes the proof.

The following two theorems demonstrate that every Bishop-Phelps cone of a reflexive Banach space can be characterized by Assumption 2.5.

Theorem 3.4. Let $\mathbb{X}$ be a reflexive Banach space, $\left(x^{*}, \alpha\right) \in X^{*} \times R_{+}$with $\alpha>0$, and let

$$
\mathbb{C}\left(x^{*}, \alpha\right)=\left\{x \in \mathbb{X}: x^{*}(x)-\alpha\|x\| \geq 0\right\}
$$

be a Bishop-Phelps cone in $\mathbb{X}$. Then $\mathbb{C}\left(x^{*}, \alpha\right)$ satisfies Assumption 2.5 with the pair $\left(x^{*}, \alpha\right)$. 
Proof. Let $x^{*} \in X^{*}$ and let $\alpha>0$ be a real number, and let $\mathbb{C}\left(x^{*}, \alpha\right)$ be a given Bishop-Phelps cone in $\mathbb{X}$. Show that $\mathbb{C}\left(x^{*}, \alpha\right)$ satisfies Assumption 2.5.

It is clear that the norm-base $\mathbb{C}_{\mathbb{U}}$ of $\mathbb{C}\left(x^{*}, \alpha\right)$ can be represented as

$$
\mathbb{C}_{\mathbb{U}}=\left\{x \in \mathbb{U}: x^{*}(x)-\alpha\|x\| \geq 0\right\}=\left\{x \in \mathbb{U}: x^{*}(x)-\alpha \geq 0 .\right\}
$$

Let

$$
\widetilde{\mathbb{C}}=\operatorname{cl}\left(\operatorname{co}\left(\mathbb{C}_{\mathbb{U}}\right)\right)
$$

As $\alpha>0$, in particular, it follows from the definition that $\mathbb{C}\left(x^{*}, \alpha\right)$ is convex and pointed.

We define the following set

$$
\mathbb{Z}=\left\{x \in \mathbb{B}: x^{*}(x) \geq \alpha .\right\}
$$

First we show that

$$
\operatorname{co}\left(\mathbb{C}_{U}\right)=\mathbb{Z}
$$

Let $x \in \operatorname{co}\left(\mathbb{C}_{U}\right)$. Then, by definition of convex hull, there exists a set of nonnegative numbers $\beta_{i}, i \in I$ such that, $x$ can be represented as

$$
x=\sum_{i \in I} \beta_{i} x_{i}, \quad \text { where } x_{i} \in \mathbb{C}_{U} \text { and } \sum_{i \in I} \beta_{i}=1 .
$$

Clearly, $x \in \mathbb{B}$. On the other hand

$$
x^{*}(x)=\sum_{i \in I} \beta_{i} x^{*}\left(x_{i}\right) \geq \alpha .
$$

Then, from $(15)$ we have $x \in \mathbb{Z}$; that is, $\operatorname{co}\left(\mathbb{C}_{U}\right) \subset \mathbb{Z}$.

Now, let $x \in \mathbb{Z}$. We will show that $x \in \operatorname{co}\left(\mathbb{C}_{U}\right)$.

If $\|x\|=1$ then $x \in \mathbb{U}$ and the inclusion $x \in \mathbb{C}_{U} \subset \operatorname{co}\left(\mathbb{C}_{U}\right)$ follows from (13).

Consider the case $\|x\|<1$, that is $x \in \operatorname{int} \mathbb{B}$. Denote $\nu=x^{*}(x)$. Clearly $\nu \geq \alpha$. Take any non-zero vector $b \in \mathbb{X}$ satisfying $x^{*}(b)=0$. Consider

$$
x_{\lambda}=x+\lambda b, \quad \lambda \in(-\infty, \infty) .
$$


We have

$$
x^{*}\left(x_{\lambda}\right)=x^{*}(x)+\lambda x^{*}(b)=\nu \geq \alpha .
$$

As $b \neq \mathbf{0}$, we have $\left\|x_{\lambda}\right\| \rightarrow \infty$ if $|\lambda| \rightarrow \infty$ which means that $x \notin \mathbb{B}$ for large values of $\lambda$. On the other hand, since $x \in \operatorname{int} \mathbb{B}$, the inclusion $x_{\lambda} \in \operatorname{int} \mathbb{B}$ holds for sufficiently small in absolute value numbers $\lambda>0$ and $\lambda<0$. Then, since $\left\|x_{\lambda}\right\|$ is weakly upper semicontinuous function of $\lambda$, and $\mathbb{B}$ is weakly compact, there are numbers $\lambda_{1}>0$ and $\lambda_{2}<0$ such that the corresponding points $x_{1} \doteq x_{\lambda_{1}}$ and $x_{2} \doteq x_{\lambda_{2}}$ belong to the boundary of $\mathbb{B}$ (as maximum values of $\left.\left\|x_{\lambda}\right\|\right)$ w.r.t. $\lambda>0$ and $\lambda<0$ respectively. That is,

$$
x_{i} \in \mathbb{U}, \quad i=1,2 \text {. }
$$

These inclusions together with (17) and (13) imply that $x_{i} \in \mathbb{C}_{U}, \quad i=1,2$.

Finally, denoting $\lambda^{\prime}=\lambda_{1} /\left(\lambda_{1}-\lambda_{2}\right)$, it is not difficult to check that,

$$
\lambda^{\prime} \in(0,1) \text { and } x=\left(1-\lambda^{\prime}\right) x_{1}+\lambda^{\prime} x_{2} .
$$

Therefore, $x \in \operatorname{co}\left(\mathbb{C}_{U}\right)$, which means that $\mathbb{Z} \subset \operatorname{co}\left(\mathbb{C}_{U}\right)$.

Thus, we have shown that the relation (16) is true. From this relation, we have

$$
\widetilde{C}=\left\{x \in \mathbb{B}: x^{*}(x) \geq \alpha\right\},
$$

and the proof is completed.

Theorem 3.5. Let $\mathbb{X}$ be a reflexive Banach space and $\mathbb{C}$ be a nonempty closed convex cone of $\mathbb{X}$. Assume that $\mathbb{C}$ satisfies Assumption 2.5 with the pair $\left(x^{*}, \alpha\right) \in X^{*} \times R_{+}$where $\alpha>0$. Then $\mathbb{C}=D\left(x^{*}, \alpha\right)$, where $D\left(x^{*}, \alpha\right)=$ $\left\{x \in \mathbb{X}: x^{*}(x)-\alpha\|x\| \geq 0\right\}$ is a Bishop-Phelps cone.

Proof. Let $x^{*} \in X^{*}$ and let $\alpha>0$ be the given pair of elements such that

$$
\operatorname{cl}\left(\operatorname{co}\left(\mathbb{C}_{\mathbb{U}}\right)\right)=\left\{x \in \mathbb{B}: x^{*}(x) \geq \alpha\right\} .
$$


Let $D\left(x^{*}, \alpha\right)=\left\{x \in \mathbb{X}: x^{*}(x)-\alpha\|x\| \geq 0\right\}$ be a BP cone formed using the same pair of elements $\left(x^{*}, \alpha\right)$. We will show that $C=D\left(x^{*}, \alpha\right)$.

Let $x \in \mathbb{C}$. Then there exists a positive real number $\beta$ such that $\beta x \in \mathbb{C}_{\mathbb{U}}$, and hence $\beta x \in \operatorname{cl}\left(\operatorname{co}\left(\mathbb{C}_{\mathbb{U}}\right)\right)$. Then by Assumption 2.5

$$
x^{*}(\beta x) \geq \alpha,
$$

and since $\beta x \in \mathbb{C}_{\mathbb{U}}$, we have $\alpha=\alpha\|\beta x\|$, and $x^{*}(\beta x) \geq \alpha\|\beta x\|$. Thus, $x^{*}(x) \geq$ $\alpha\|x\|$, which means that $x \in D\left(x^{*}, \alpha\right)$.

Now let $x \in D\left(x^{*}, \alpha\right) \backslash\{0\}$. Then $x /\|x\| \in \mathbb{U}$, and therefore $x^{*}(x /\|x\|) \geq$ $\alpha$. By Assumption $2.5 x /\|x\| \in \mathbb{U}$, which implies that $x /\|x\| \in \operatorname{cl}\left(\operatorname{co}\left(\mathbb{C}_{\mathbb{U}}\right)\right)$. Then there exists a sequence $\left\{y_{n}\right\} \subset \operatorname{co}\left(\mathbb{C}_{\mathbb{U}}\right)$ such that $y_{n} \rightarrow x /\|x\|$. By the convexity of $\mathbb{C}$ we have $y_{n} \in \mathbb{C}$, and by the closedness of $\mathbb{C}$ we obtain that $x /\|x\| \in \mathbb{C}$.

The following example illustrates the separation property, Lemma 3.3 and Theorem 3.5.

Example 3.6. Let $X=\mathbb{R}^{2}, \mathbb{C}=\{(s, s): s \geq 0\}$ and $\mathbb{K}=\{(s, t): s \leq$ 0 or $t \leq 0\}$. Since the separation property depends on the norm, we will investigate this example with respect to three norms in $\mathbb{R}^{2}$.

Let $\|\cdot\|_{1},\|\cdot\|_{2}$ and $\|\cdot\|_{\infty}$ be the $l_{1}, l_{2}$ and $l_{\infty}$ norms respectively, where $\|x\|_{1}=|s|+|t|,\|x\|_{2}=\sqrt{s^{2}+t^{2}}$, and $\|x\|_{\infty}=\max \{|s|,|t|\}$, for every $x=$ $(s, t) \in \mathbb{R}^{2}$.

For $x^{*}=(1,1)$, it is easy to calculate $\alpha=\max \left\{x^{*}\left(\frac{x}{\|x\|}\right): x \in \mathbb{R}^{2}.\right\}$ It is clear that $\alpha_{1}=1, \alpha_{2}=\sqrt{2}$, and $\alpha_{\infty}=2$, where $\alpha_{1}, \alpha_{2}$ and $\alpha_{\infty}$ are the maximum values of $\alpha$ calculated with respect to $l_{1}, l_{2}$ and $l_{\infty}$ norms respectively.

Let $\mathbb{C}_{U_{1}}, \mathbb{C}_{U_{2}}$ and $\mathbb{C}_{U_{\infty}}$ be the norm-bases of $\mathbb{C}$ w.r.t. $l_{1}, l_{2}$ and $l_{\infty}$ norms respectively.

Then, we have $\tilde{\mathbb{C}}_{1}=\operatorname{cl}\left(\operatorname{co}\left(\mathbb{C}_{U_{1}}\right)\right)=\{(1 / 2,1 / 2)\}$, and for $x^{*}=(1,1)$ and $\alpha_{1}=1$ one has $\left\{x \in B: x^{*}(x) \geq \alpha_{1}\right\}=\{(s, t): s+t=1, s \geq 0, t \geq 0\}$, hence $\operatorname{cl}\left(\operatorname{co}\left(\mathbb{C}_{U_{1}}\right)\right) \neq\left\{x \in B: x^{*}(x) \geq \alpha_{1}\right\}$. Therefore, Assumption 2.5 is not satisfied for $\mathbb{C}$ (for the case of $l_{1}$ norm). Noting that $\mathbb{K}_{U_{1}}^{\partial}=\mathbb{K}_{U_{2}}^{\partial}=$ $\mathbb{K}_{U_{\infty}}^{\partial}=\{(0,1),(1,0)\}$, one has $\widetilde{\mathbb{K}}^{\partial}=\{(s, t): s \geq 0, t \geq 0$ and $s+t \leq 1\}$. Since $\widetilde{\mathbb{K}}^{\partial} \cap \tilde{\mathbb{C}}_{1}=\{(1 / 2,1 / 2)\} \neq \emptyset$, the separation property is not satisfied 
for these cones. Finally, since Assumption 2.5 is not satisfied, $\mathbb{C}$ must not be a Bishop-Phelps cone by Theorem 3.5. Indeed, there does not exist a pair $\left(x^{*}, \alpha\right)$ with $\mathbb{C}=\left\{x=(s, t): x^{*}(x) \geq \alpha\|x\|\right\}$, and the smallest BishopPhelps cone containing $\mathbb{C}$ is a cone of the form $\{(s, t): s+t \geq|s|+|t|\}$ (with $\left.x^{*}=(1,1), \alpha_{1}=1\right)$ which equals $R_{+}^{2}$.

On the other hand, $\tilde{\mathbb{C}}_{2}=\operatorname{cl}\left(\operatorname{co}\left(\mathbb{C}_{U_{2}}\right)\right)=\left\{x \in B: x^{*}(x) \geq \alpha_{2}\right\}=$ $\{(\sqrt{2} / 2, \sqrt{2} / 2)\}$. Hence $\mathbb{C}$ satisfies Assumption 2.5 for $l_{2}$ norm, and because of $\mathbb{C} \cap \mathbb{K}=\{0\}$, by Lemma 3.3 the cones $\mathbb{C}$ and $\mathbb{K}$ must satisfy the separation property given in Definition 3.1. We have $\widetilde{\mathbb{K}}^{\partial} \cap \tilde{\mathbb{C}}=\emptyset$ and hence $\mathbb{C}$ and $\mathbb{K}$ satisfy the separation property given in Definition 3.1.

Note also that, since $\mathbb{C}$ satisfies Assumption 2.5, by Theorem 3.5 it must be represented as a Bishop-Phelps cone. Indeed, for $x^{*}=(1,1)$ and $\alpha_{2}=\sqrt{2}$ we have

$$
\mathbb{C}=\left\{x=(s, t): x^{*}(x) \geq \alpha_{2}\|x\|_{2}\right\}=\left\{(s, t): s+t \geq \sqrt{2} \sqrt{s^{2}+t^{2}} \cdot\right\}
$$

Finally, it can be shown in a similar way that $\tilde{\mathbb{C}}_{\infty}=\operatorname{cl}\left(\operatorname{co}\left(\mathbb{C}_{U_{\infty}}\right)\right)=\{x \in$ $\left.B: x^{*}(x) \geq \alpha_{\infty}\right\}=\{(1,1)\}$. Hence $\mathbb{C}$ satisfies Assumption 2.5 and the separation property together with the cone $\mathbb{K}$ for the case of $l_{\infty}$ norm, and $\mathbb{C}$ can be represented as a Bishop-Phelps cone:

$$
\mathbb{C}=\left\{x=(s, t): x^{*}(x) \geq \alpha_{\infty}\|x\|_{\infty}\right\}=\{(s, t): s+t \geq 2 \max \{|s|,|t|\} .\}
$$

The following two theorems proved in [18] concern the existence of a pair $\left(x^{*}, \alpha\right) \in \mathbb{C}^{a \#}$ for which the corresponding sublevel set $S\left(x^{*}, \alpha\right)$ of the strongly monotonically increasing sublinear function $g(x)=x^{*}(x)+\alpha\|x\|$ separates the given cones $\mathbb{C}$ and $\mathbb{K}$, where $S\left(x^{*}, \alpha\right)$ is defined as

$$
S\left(x^{*}, \alpha\right)=\left\{x \in \mathbb{X}: x^{*}(x)+\alpha\|x\| \leq 0 .\right\}
$$

Theorem 3.7. Let $\mathbb{C}$ and $\mathbb{K}$ be closed cones in a reflexive Banach space $(\mathbb{X},\|\cdot\|)$. Assume that the cones $-\mathbb{C}$ and $\mathbb{K}$ satisfy the separation property defined in Definition 3.1,

$$
-\widetilde{\mathbb{C}} \cap \widetilde{\mathbb{K}}^{\partial}=\emptyset,
$$


Then, $\mathbb{C}^{a \#} \neq \emptyset$, and there exists a pair $\left(x^{*}, \alpha\right) \in \mathbb{C}^{a \#}$ such that the corresponding sublevel set $S\left(x^{*}, \alpha\right)$ of the strongly monotonically increasing sublinear function $g(x)=x^{*}(x)+\alpha\|x\|$ separates the cones $-\mathbb{C}$ and $b d(\mathbb{K})$ in the following sense:

$$
x^{*}(x)+\alpha\|x\|<0 \leq x^{*}(z)+\alpha\|z\|
$$

for all $x \in-\mathbb{C} \backslash\left\{0_{\mathbb{X}}\right\}$, and $z \in b d(\mathbb{K})$. In this case the cone $-\mathbb{C}$ is pointed.

Conversely, if there exists a pair $\left(x^{*}, \alpha\right) \in \mathbb{C}^{a \#}$ such that the corresponding sublevel set $S\left(x^{*}, \alpha\right)$ of the strongly monotonically increasing sublinear function $g(x)=x^{*}(x)+\alpha\|x\|$ separates the cones $-\mathbb{C}$ and bd $(\mathbb{K})$ in the sense of (20) and if either the cone $\mathbb{C}$ is closed and convex or $(\mathbb{X},\|\cdot\|)$ is a finite dimensional space, then the cones $-\mathbb{C}$ and $\mathbb{K}$ satisfy the separation property (19).

Theorem 3.8. Let $\mathbb{C}$ be a closed cone of a reflexive Banach space $\left(\mathbb{Y},\|\cdot\|_{\mathbb{Y}}\right)$, and let $\mathbb{C}_{\varepsilon}$ be its $\varepsilon$-conic neighborhood for a real number $\varepsilon \in(0,1)$. Suppose that $\mathbb{C}$ and $\mathbb{C}_{\varepsilon}$ satisfy the separation property given in Definition 3.1. Then, there exists a pair $\left(y^{*}, \alpha\right) \in \mathbb{C}^{a \#}$ such that

$$
-\mathbb{C} \backslash\left\{0_{\mathbb{Y}}\right\} \subset \operatorname{int}\left(S\left(y^{*}, \alpha\right)\right) \subset-\mathbb{C}_{\varepsilon},
$$

where $\operatorname{int}\left(S\left(y^{*}, \alpha\right)\right)$ can be defined as

$$
\operatorname{int}\left(S\left(y^{*}, \alpha\right)\right)=\left\{y \in \mathbb{Y}: y^{*}(y)+\alpha\|y\|<0\right\} .
$$

\section{The main result}

In this section we formulate the generalization of Arrow-Barankin- Blackwell (ABB) theorem for nonconvex sets of reflexive Banach spaces partially ordered by a Bishop-Phelps cone.

First we present the following lemma without proof which gives important properties of the so-called dilating cones $\mathbb{C}_{\varepsilon}$ constructed by using the normbase $\mathbb{C}_{U}$ of $\mathbb{C}$. Its proof is obvious and similar to the proof of lemmas given for Henig dilating cones in the literature; see for example [16, 8, 14]. 
Lemma 4.1. Let $\mathbb{C} \subset \mathbb{X}$ be a closed convex cone. For $\varepsilon>0$ let

$$
\mathbb{C}_{\varepsilon}=\operatorname{cone}\left(\mathbb{C}_{U}+\varepsilon \mathbb{B}\right)
$$

be the dilating cone. Then

(i) $\mathbb{C}_{\varepsilon}$ is a closed cone for every $\varepsilon>0$;

(ii) $\mathbb{C} \backslash\{0\} \subset \mathbb{C}_{\varepsilon_{1}} \backslash\{0\} \subset \operatorname{int}\left(\mathbb{C}_{\varepsilon_{2}}\right)$ for all $0<\varepsilon_{1}<\varepsilon_{2}$;

(iii) For a sequence of positive numbers $\varepsilon_{n}$ converging to zero, the sequence of cones $\left\{\mathbb{C}_{\varepsilon_{n}}\right\}$ converges to $\mathbb{C}$ in the following sense: for every positive $\varepsilon$ there exists a positive integer $\mathbb{N}$ such that all cones $\mathbb{C}_{n}$ with $n>\mathbb{N}$ are in $\varepsilon$ conic neighborhood of $\mathbb{C}$.

Theorem 4.2. Let $\left(\mathbb{X},\|\cdot\|_{\mathbb{X}}\right)$ be a reflexive Banach space partially ordered by a Bishop-Phelps cone $\mathbb{C}$. Assume that $\mathbb{S}$ is a nonempty set of $\mathbb{X}$ and there exists a positive scalar $\delta$ such that $\mathbb{S}$ is Hartley $\mathbb{C}_{\delta}$-compact, where $\mathbb{C}_{\delta}$ is the $\delta$ - neighborhood of $\mathbb{C}$. Let $\bar{x} \in \mathbb{S}$ be a minimal element of $\mathbb{S}$. Then for every $\varepsilon \in(0,1)$ there exists a proper efficient point $x_{\varepsilon}$ of $\mathbb{S}$ such that

$$
\left\|x_{\varepsilon}-\bar{x}\right\| \leq \varepsilon \text {. }
$$

Proof. Let $\bar{x} \in \mathbb{S}$ be a minimal element of $\mathbb{S}$, and let $\varepsilon \in(0,1)$ be a given number. Let $\varepsilon_{n}$ be a sequence of decreasing positive numbers converging to zero, and let

$$
\mathbb{C}_{n}=\operatorname{cone}\left(\mathbb{C}_{\mathbb{U}}+\varepsilon_{n} \mathbb{B}\right)
$$

be dilating cone, where $\mathbb{C}_{\mathbb{U}}$ is a norm-base of $\mathbb{C}$. Then, it is clear that $\mathbb{C}_{n}$ is a closed cone, $\mathbb{C} \backslash\{0\} \subset \operatorname{int}\left(\mathbb{C}_{n}\right)$ and $\mathbb{C}_{n+1} \backslash\{0\} \subset \operatorname{int}\left(\mathbb{C}_{n}\right)$ for every $n$, and the sequence of cones $\left\{\mathbb{C}_{n}\right\}$ converges to $\mathbb{C}$ (see Lemma 4.1).

Note also that, since $\mathbb{S}$ has been assumed to be closed and $\mathbb{C}_{\delta}$-compact, it is $\mathbb{C}_{n}$-compact for every $n>n_{\delta}$, where $n_{\delta}=\min \left\{n: \varepsilon_{n}<\delta\right.$. $\}$

The proof of the theorem consists of two parts.

1. First we will prove that for the given positive scalar $\varepsilon$, there exists an integer $n_{\varepsilon}$ such that

$$
\left(\{\bar{x}\}-\mathbb{C}_{n}\right) \cap \mathbb{S} \subset(\{\bar{x}\}+\varepsilon \mathbb{B}) \cap \mathbb{S},
$$

for all $n \geq n_{\varepsilon}$. 
Suppose that this is not true. Then, there exists a scalar $\gamma>0$ such that, for every $n>n_{\delta}$, one can find $n_{\gamma}>n$ with

$$
\left(\{\bar{x}\}-\mathbb{C}_{n_{\gamma}}\right) \cap \mathbb{S} \nsubseteq(\{\bar{x}\}+\gamma \mathbb{B}) \cap \mathbb{S} .
$$

Hence, for every $n>n_{\delta}$ there exist $x_{n} \in\left(\{\bar{x}\}-\mathbb{C}_{n}\right) \cap \mathbb{S}$ with $\left\|x_{n}-\bar{x}\right\|>\gamma$. Because of the compactness of $\left(\bar{x}-\mathbb{C}_{n}\right) \cap \mathbb{S}$, the sequence $\left\{x_{n}\right\}$ contains convergent subsequence $\left\{x_{n_{k}}\right\}$. Let $x_{n_{k}} \rightarrow x_{0}$. Then, from relations $x_{n_{k}} \in$ $\left(\{\bar{x}\}-\mathbb{C}_{n_{k}}\right) \cap \mathbb{S}$ and $\left\|x_{n_{k}}-\bar{x}\right\|>\gamma$ we obtain $x_{0} \in(\{\bar{x}\}-\mathbb{C}) \cap \mathbb{S}$ and $x_{0} \neq \bar{x}$, which contradicts to the hypothesis that $\bar{x}$ is a minimal element of $\mathbb{S}$. Thus, the relation (24) has been proved.

2. Denote $\mathbb{C}_{\varepsilon}=\mathbb{C}_{n_{\varepsilon}}$. Since the ordering cone $\mathbb{C}$ is a Bishop-Phelps cone, by Theorem 3.4 it satisfies Assumption 2.5. Then by Lemma 3.3, cones $\mathbb{C}$ and $\mathbb{C}_{\varepsilon}$ satisfy the separation property. Therefore by Theorem 3.7 , there exists a pair $\left(x_{\varepsilon}^{*}, \alpha_{\varepsilon}\right) \in \mathbb{C}^{a \#}$ such that the corresponding sublevel set $\mathbb{S}\left(x_{\varepsilon}^{*}, \alpha_{\varepsilon}\right)$ of the strongly monotonically increasing sublinear function $g(x)=x_{\varepsilon}^{*}(x)+\alpha_{\varepsilon}\|x\|$ separates the cones $-\mathbb{C}$ and $-b d\left(\mathbb{C}_{\varepsilon}\right)$ in the following sense:

$$
x_{\varepsilon}^{*}(x)+\alpha_{\varepsilon}\|x\|<0 \leq x_{\varepsilon}^{*}(z)+\alpha_{\varepsilon}\|z\|
$$

for all $x \in-\mathbb{C} \backslash\left\{0_{\mathbb{X}}\right\}$, and $z \in-\mathrm{bd}\left(\mathbb{C}_{\varepsilon}\right)$.

Note that

$$
\mathbb{S}\left(x_{\varepsilon}^{*}, \alpha_{\varepsilon}\right)=\left\{x \in \mathbb{X}: x_{\varepsilon}^{*}(x)+\alpha_{\varepsilon}\|x\| \leq 0,\right\}
$$

and

$$
\operatorname{int}\left(\mathbb{S}\left(x_{\varepsilon}^{*}, \alpha_{\varepsilon}\right)\right)=\left\{x \in \mathbb{X}: x_{\varepsilon}^{*}(x)+\alpha_{\varepsilon}\|x\|<0\right\}
$$

(see [18, Lemma 3.6]).

Let

$$
\mathbb{S}_{\varepsilon}=\left(\bar{x}-\mathbb{C}_{\varepsilon}\right) \cap \mathbb{S} .
$$

It follows from (24) that

$$
\mathbb{S}_{\varepsilon} \subset(\{\bar{x}\}+\varepsilon \mathbb{B}) \cap \mathbb{S},
$$

Then it is clear that (see Theorem 3.8)

$$
-\mathbb{C} \backslash\{0\}_{\mathbb{X}} \subset \operatorname{int}\left(\mathbb{S}\left(x_{\varepsilon}^{*}, \alpha_{\varepsilon}\right)\right) \subset-\mathbb{C}_{\varepsilon}
$$


and

$$
\left(\{\bar{x}\}+\mathbb{S}\left(x_{\varepsilon}^{*}, \alpha_{\varepsilon}\right)\right) \cap \mathbb{S} \subset \mathbb{S}_{\varepsilon}
$$

Since $g(x)=x_{\varepsilon}^{*}(x)+\alpha_{\varepsilon}\|x\|$ is weakly lower semicontinuous, and $\mathbb{S}_{\varepsilon}$ is compact, $g$ achieves its minimum on $\mathbb{S}_{\varepsilon}$. Thus, there exists $x_{\varepsilon} \in \mathbb{S}_{\varepsilon}$ such that

$$
x_{\varepsilon}^{*}\left(x_{\varepsilon}\right)+\alpha_{\varepsilon}\left\|x_{\varepsilon}\right\| \leq x_{\varepsilon}^{*}(x)+\alpha_{\varepsilon}\|x\| \text { for all } x \in \mathbb{S}_{\varepsilon},
$$

or

$$
x_{\varepsilon}^{*}\left(x-x_{\varepsilon}\right)+\alpha_{\varepsilon}\left\|x-x_{\varepsilon}\right\| \geq 0 \text { for all } x \in \mathbb{S}_{\varepsilon} .
$$

This means by Definition 2.4 that $x_{\varepsilon}$ is a proper minimal element of $\mathbb{S}_{\varepsilon}$ and in the case that $x_{\varepsilon}$ is the unique minimum of $g$ on $\mathbb{S}_{\varepsilon}$, the following relation holds:

$$
\left(\left\{x_{\varepsilon}\right\}+\mathbb{S}\left(x_{\varepsilon}^{*}, \alpha_{\varepsilon}\right)\right) \cap \mathbb{S}_{\varepsilon}=\left\{x_{\varepsilon}\right\} .
$$

If $x_{\varepsilon}$ is not a unique minimum of $g$ on $\mathbb{S}_{\varepsilon}$, then the above intersection may also contain other minimums. In this case, because of the inclusions

$$
-\mathbb{C} \backslash\{0\}_{\mathbb{X}} \subset \operatorname{int}\left(\mathbb{S}\left(x_{\varepsilon}^{*}, \alpha_{\varepsilon}\right)\right) \subset-\mathbb{C}_{\varepsilon},
$$

for some $\bar{\varepsilon}>0$ there exists a dilating cone $\mathbb{C}_{\bar{\varepsilon}}$ such that

$$
-\mathbb{C}_{\bar{\varepsilon}} \backslash\{0\}_{\mathbb{X}} \subset \operatorname{int}\left(\mathbb{S}\left(x_{\varepsilon}^{*}, \alpha_{\varepsilon}\right)\right) .
$$

Then, it is clear that

$$
\left(\left\{x_{\varepsilon}\right\}-\mathbb{C}_{\bar{\varepsilon}}\right) \cap \mathbb{S}_{\varepsilon}=\left\{x_{\varepsilon}\right\} .
$$

Now we show that $x_{\varepsilon}$ is a properly minimal element of the entire set $\mathbb{S}$. Assume to the contrary that this is not true: there exists $\widehat{x} \in \mathbb{S} \backslash\left\{x_{\varepsilon}\right\}$ such that

$$
\widehat{x} \in\left(\left\{x_{\varepsilon}\right\}-\mathbb{C}_{\bar{\varepsilon}}\right) \cap \mathbb{S} .
$$

It is clear that $\widehat{x} \notin \mathbb{S}_{\varepsilon}$. Then, since

$$
-\mathbb{C}_{\bar{\varepsilon}} \backslash\{0\}_{\mathbb{X}} \subset \operatorname{int}\left(\mathbb{S}\left(x_{\varepsilon}^{*}, \alpha_{\varepsilon}\right)\right),
$$

and $\mathbb{S}\left(x_{\varepsilon}^{*}, \alpha_{\varepsilon}\right) \subset-\mathbb{C}_{\varepsilon}$, we obtain (see $(26)$ )

$$
\widehat{x} \in\left(\{\bar{x}\}-\mathbb{C}_{\varepsilon}\right) \cap \mathbb{S}=\mathbb{S}_{\varepsilon},
$$

which is a contradiction. Hence, it has been proved that $x_{\varepsilon}$ is a proper minimal element of the entire set $\mathbb{S}$. Then since $x_{\varepsilon} \in \mathbb{S}_{\varepsilon}$, the proof of the theorem follows now from (27). 
Remark 4.3. The following example demonstrates that the Hartley $\mathbb{C}_{\delta}$-compactness condition of $\mathbb{S}$ cannot be weakened to Hartley $\mathbb{C}$-compactness condition.

\section{Example 4.4. Let}

$$
\mathbb{S}=\left\{x=\left(x_{1}, x_{2}\right) \in \mathbb{R}^{2}: x_{1}<0, x_{2}<0, x_{2} \geq 1 / x_{1}\right\},
$$

and $\mathbb{C}=\mathbb{R}_{+}^{2}$. Then $\mathbb{S}$ is Hartley $\mathbb{C}$-compact, but is not Hartley $\mathbb{C}_{\delta}$-compact for every $\delta>0$. The set of efficient points of $\mathbb{S}$ consists of the whole boundary curve:

$$
\left\{x=\left(x_{1}, x_{2}\right) \in \mathbb{R}^{2}: x_{1}<0, x_{2}<0, x_{2}=1 / x_{1}\right\} .
$$

The set of properly efficient points of $\mathbb{S}$ is empty, and hence the assertion of $A B B$ Theorem is not true for this case.

Remark 4.5. It is well-known that if $\mathbb{S}$ is asymptotically compact with $\mathbb{S}_{\infty} \cap$ $\mathbb{C}=\{0\}$, then $\mathbb{S}$ is Hartley $\mathbb{C}$-compact (see [13, Proposition 3.2.26]). But the inverse conclusion is proved only for convex sets $\mathbb{S}$. Therefore, Example 4.4 demonstrates that the density result obtained in [14] does not cover the result presented in this paper.

\section{Conclusions}

In this paper, we present a generalization of Arrow-Barankin-Blackwell theorem for properly efficient points defined as support points of sets with respect to monotonically increasing sublinear functions. This theorem is shown to hold for Hartley cone compact sets of reflexive Banach spaces partially ordered by a Bishop-Phelps cone. A characterization of Bishop-Phelps cones in the form of necessary and sufficient condition is also presented. 


\section{References}

[1] K. J. Arrow, E. W. Barankin, and D. Blackwell, Admissible points of convex sets, in Contribution to the Theory of Games, H. W. Kuhn and A. W. Tucker, eds., Princeton University Press, Princeton, NJ, 1953

[2] E. M. Bednarczuk, And W. Song, Some More Density Results for Proper Efficiency, Journal of Mathematical Analysis and Applications, 231, pp. 345-354 (1999).

[3] H.P. Benson, An improved definition of proper efficiency for vector maximization with respect to cones, J. Math. Anal. Appl., 71, pp. 232241 (1979).

[4] G.R. Bitran and T.L. Magnanti, The Structure of Admissible Points with Respect to Cone Dominance, Journal of Optimization Theory and Applications, 29, pp. 573-614 (1979).

[5] J.M. Borwein, Proper Efficient Points for Maximization with Respect to Cones, SIAM Journal on Control and Optimization, 15, pp. 57-63 (1977).

[6] J.M. Borwein, On the Existence of Pareto Efficient Points, Mathematics of Operation Research,8, pp. 64-73 (1980).

[7] J.M. Borwein, The geometry of Pareto efficiency over cones, Mathematische Operationsforschung und Statistik, Series Optimization, 11, pp. 235-248 (1980).

[8] J.M. Borwein And D. Zhuang, Super Efficiency in Vector Optimization, Transactions of the American Mathematical Society, 338, pp. 105122 (1993).

[9] F. Ferro, A new ABB theorem in Banach spaces, Optimization 46, pp. 353-362 (1999).

[10] W.T. Fu, On the Density of Proper Efficient Points, Proceedings of the American Mathematical Society, 124, pp. 1213-1217 (1996). 
[11] R.J. Gallagher and O.A. SAleh, Two Generalizations of a Theorem of Arrow, Barankin, and Blackwell, SIAM Journal on Control and Optimization, 31, pp. 247-256 (1993).

[12] R. N. Gasimov, Characterization of the Benson proper efficiency and scalarization in nonconvex vector optimization, in: M.Koksalan, S.Zionts (eds.) Multiple Criteria Decision Making in the New Millennium, Book Series: Lecture Notes in Econom. and Math. Systems, 507, pp. 189-198 (2001).

[13] A. Gopfert, Riahi, Chr. Tammer and C. Zalinescu, Variational Methods in Partially Ordered Spaces, Springer-Verlag-New York (2003).

[14] A. Gopfert, Chr. Tammer and C. Zalinescu, A new ABB theorem in normed vector spaces, Optimization, 53, No4, pp. 369-376 (2004).

[15] R. Hartley, On Cone Efficiency, Cone Conexity, and Cone Compactness, SIAM Journal on Applied Mathematics, 34, pp. 211-222 (1978).

[16] M. I. Henig, Proper efficiency with respect to cones, J. Optim. Theory Appl., 36, pp. 387-407 (1982).

[17] J. JAHn, A generalizationof a theorem of Arrow, Barankin, and Blackwell, SIAM J. Control and Optimization, 26, pp. 999-1005 (1988).

[18] R. Kasimbeyli, A Nonlinear Cone Separation Theorem and Scalarization in Nonconvex Vector Optimization, SIAM J. on Optimization, 20, pp. 1591-1619 (2010).

[19] R. Kasimbeyli, A conic scalarization method in multi-objective optimization, Journal of Global Optimization, in press, DOI: 10.1007/s10898-011-9789-8.

[20] D. T. Luc, Theory of Vector Optimization, Lecture Notes in Econom. and Math. Systems 319, Springer-Verlag, Berlin (1989).

[21] M. Majumdar, Some approximation theorems on efficiency prices for infinite programs, Journal of Economic Theory, 2, pp. 399-410(1970). 
[22] M. Majumdar, Some General Theorems on Efficiency Prices with an Infinite-Dimensional Commodity Space, Journal of Economic Theory, 5, pp. 1-13 (1972).

[23] E.K. Makarov and N.N. Rachkovski, Density Theorem for Generalized Henigs Proper Efficiency, Journal of Optimization Theory and Applications, 91, pp. 419-437 (1996).

[24] K.F Ng And X.Y. Zheng, On the Density of Positive Proper Efficient Points in a Normed Space, JOTA, 119, pp. 105-122 (2003).

[25] R. RAdner, A note on maximal points of convex sets in $l_{\infty}$, in Proc. of the Fifth Berkeley Symposium on Mathematical Statistics and Probability, L.M. Le Cam and J. Neyman, eds., University of California Press, Berkeley, CA (1967).

[26] W. SALz, Eine topologische eigenschaft der effizienten punkte konvexer mengen, Operat. Res. Verfahren, XXIII, (1976), pp. 197-202.

[27] X.D.H. Truong, Existence and density results for proper efficiency in cone compact sets, Journal of Optimization Theory and Applications, 111, pp. 173-194 (2001).

[28] L.W. Woo And R.K. Goodrich, Maximal Points of Convex Sets in Locally Convex Topological Vector Spaces: Generalization of the ArrowBarankin-Blackwell Theorem, JOTA, 116, pp. 647-658 (2003).

[29] X.Y. Zheng, Proper Efficiency in Locally Convex Topological Vector Spaces, Journal of Optimization Theory and Applications, 94, pp. 469486 (1997).

[30] X.Y. Zheng, The Domination Property for Efficiency in Locally Conex Topological Vector Spaces, Journal of Mathematical Analysis and Applications, 213, pp. 455-467 (1997).

[31] X.Y. Zheng, Generalizations of a Theorem of Arrow, Barankin, and Blackwell in Topological Vector Spaces, JOTA, 96, pp. 221-233 (1998).

[32] D. Zhuang, Density Results for Proper Efficiency, SIAM Journal on Control and Optimization, 32, pp. 51-58 (1994). 